\title{
Intent For Diagnosis
}

National Cancer Institute

\section{Source}

National Cancer Institute. Intent For Diagnosis. NCI Thesaurus. Code C54415.

The use of a particular medical device, medication or treatment to help establish the presence or absence of a disease or condition. 